\title{
The Added Burden of Transfer Status in Patients Undergoing Surgery After Sustaining a Periprosthetic Fracture of the Hip or Knee
}

\author{
Edwin Chaharbakhshi ${ }^{1}$, Daniel Schmitt ${ }^{2}$, Nicholas M. Brown ${ }^{3}$ \\ 1. Orthopaedic Surgery, West Virginia University, Morgantown, USA 2. Orthopaedic Surgery, Loyola University Medical \\ Center, Maywood, USA 3. Orthopaedic Surgery \& Rehabilitation, Loyola University Medical Center, Maywood, USA
}

Corresponding author: Edwin Chaharbakhshi, eddiechaharbakhshi@gmail.com

\section{Abstract \\ Background}

A significant proportion of patients who sustain periprosthetic fractures are transferred to another institution for definitive care. There is limited understanding of the impact of transfer on outcomes.

\section{Methods}

The American College of Surgeons National Surgical Quality Improvement Program (NSQIP) was utilized to identify patients who sustained periprosthetic fractures of the hip or knee requiring surgical intervention. Inclusion criteria were total joint prosthesis of the hip or knee with documented periprosthetic fracture of a single hip or knee along with surgery during hospitalization. Transfer status and discharge location were recorded. A total of 1,194 non-transferred patients were compared to 620 transferred patients. Cohorts were compared utilizing standard parametric and non-parametric tests depending on the characteristics of the data.

\section{Results}

Transferred patients had a higher mean age (75.6 years vs. 72.9 years, $p<0.0001)$, longer mean length of stay ( 7.9 days vs. 6.9 days, $\mathrm{p}=0.0023$ ), and greater American Society of Anesthesiologists (ASA) grade. Transferred patients were less likely to be discharged home $(p=0.0001)$ and more likely to be discharged to hospice $(p=0.049)$ or rehabilitation facilities $(p=0.0001)$. No significant differences were detected regarding readmissions or complications. In transferred patients, having lower preoperative albumin was a risk factor for readmission within 30 days.

\section{Conclusion}

Transfer centers accepting and treating periprosthetic fractures should be aware that these patients often have a longer length of stay and are less likely to be discharged home. However, the data suggests these patients are well cared for, given the similar complication rates.

Review began 07/14/2021 Review ended 07/27/2021 Published 08/01/2021

\section{() Copyright 2021}

Chaharbakhshi et al. This is an open access article distributed under the terms of the Creative Commons Attribution License CC-BY 4.0., which permits unrestricted use, distribution, and reproduction in any medium, provided the original author and source are credited.
Categories: Emergency Medicine, Orthopedics, Trauma

Keywords: arthroplasty, periprosthetic fracture, transfer, readmission, complication

\section{Introduction}

Interfacility patient transfers are a significant logistical undertaking dependent on factors such as handoffs, procedures, risk stratification, and unpredictability [1,2]. There is a breadth of literature evaluating the potentially negative effects of transfer status on patient outcomes across disciplines [3-7]. A recent study by Mueller et al. reported that between $32 \%$ and $89 \%$ of transferred patients did not receive specialty care despite being transferred from an acute care hospital, suggesting that a significant proportion of transfers may not have been appropriate [3]. Overall, inappropriate patient transfers can accumulate significant burdens in cost and resource allocation to healthcare systems worldwide.

There has been a growing body of research over the past decade exploring orthopedic trauma transfers. O'Connell et al. demonstrated that up to $20 \%$ of orthopedic trauma transfers at a level I trauma center were inappropriate [8]. This observation was even larger in Thakur's study, which reported that up to $52 \%$ of orthopedic trauma transfers were inappropriate [9]. In terms of risk, a recent study by Boyd et al. demonstrated that patients with lower extremity fractures who were subsequently transferred had a $9.5 \%$ risk of developing venous thromboembolism, compared to $0.7 \%$ in non-transferred patients [10]. Importantly, some of the findings in recent literature may be related to the observation that only a limited number of patients are examined by an orthopedic surgeon prior to transfer [11]. 
Given that many periprosthetic fracture patients undergo transfer, it is important to determine whether these patients differed from non-transferred patients in terms of demographics, comorbidities, and outcomes. A more definitive answer to this question may allow physicians and health systems to better anticipate appropriate levels of care and understand the impact of treating these complex patients. We hypothesized that patients who required transfers to another facility would have more comorbidities, perioperative complications, and would be less likely to be discharged home from the hospital.

\section{Materials And Methods}

\section{Data source}

The authors of the present study requested and were granted access to the American College of Surgeons National Surgical Quality Improvement Program (NSQIP) database. This database is a nationally validated tool that has been previously used to assist in improving surgical care in general. The NSQIP database contained various data for surgical cases that occurred between 2015 and 2018. Of note, the data analysis is available for sharing in the form of a Microsoft Excel (Microsoft Corporation, Redmond, Washington, United States) and/or MiniTab spreadsheet.

\section{Study design}

The NSQIP database (cases between 2015 and 2018) was used as the sole source of data. The present study was designed as a retrospective cohort study that compared patients who sustained a single periprosthetic fracture of the hip or knee, were hospitalized, and were either transferred to a new facility or not transferred for surgical intervention of that periprosthetic fracture. Inclusion criteria were patients who had a preexisting total joint prosthesis of the hip or knee, had a documented periprosthetic fracture of the hip or knee, underwent surgical intervention during the same hospitalization, had a transfer status documented (non-transfers vs. transfers), and had a discharge location documented. Exclusion criteria were patients who had more than one affected prosthetic joint or required any other surgical interventions during the total hospitalization period.

\section{Data utilized}

Patient demographic variables, comorbidities, preoperative laboratory values, perioperative complications (stroke, myocardial infarction, pneumonia, surgical site infection, pulmonary embolism, urinary tract infection), readmissions, and discharge destination were used. Although initially requested, insurance information and specific surgical dates were not available to NSQIP users to help ensure patient confidentiality. After applying the aforementioned inclusion and exclusion criteria, the desired data was isolated into a separate file for statistical analysis.

\section{Statistical analysis}

All analyses were performed using Microsoft Excel and MiniTab. Descriptive statistics including mean, standard deviation, ranges, and proportions were calculated and reported when appropriate. The ShapiroWilk test was performed to assess continuous data for normal distributions. The F-test was performed to demonstrate which continuous data demonstrated equal variances. For data following a normal distribution, the two-tailed independent samples t-test was performed to compare mean differences between groups. If not normally distributed, the Mann-Whitney U test was utilized. The Chi-squared and Fisher's exact tests were performed to assess for differences in proportions between groups when using categorical data. A multiple linear regression was performed with forced entry (the enter method) to identify the odds of 30-day readmission based on the preoperative laboratory values housed in NSQIP. Odds ratios and confidence intervals that met the threshold for statistical significance $(\mathrm{p}<0.05)$ were reported.

\section{Results}

\section{Patient demographics}

After isolating patients who sustained periprosthetic fractures of the hip or knee, a group of 1194 patients who did not require transfer were compared to a group of 620 patients who required transfer prior to surgical intervention. Comparisons of demographics are detailed in Table 1. Patients who required transfers were, on average, older (75.6 years vs. 72.9 years, $\mathrm{p}<0.0001)$, more likely to be male $(35.0 \%$ vs. $29.3 \%, \mathrm{p}=0.0132)$, more likely to have a periprosthetic hip as compared to knee fracture $(78.1 \%$ vs. $69.6 \%, \mathrm{p}=0.0001)$, and had a longer time from the initial hospital's admission to eventual discharge (7.9 days vs. 6.9 days, $\mathrm{p}=0.0023$ ). No statistically significant differences were observed between the two groups regarding ethnicity or mean body mass index (BMI). 


\section{Cureus}

\begin{tabular}{|c|c|c|c|}
\hline Patient demographics & Non-transfers & Transfers & P-value \\
\hline Patients (n) & 1194 & 620 & \\
\hline Age in years (mean, SD) & $72.9(10.9)$ & 75.6 (10.7) & $<0.0001$ \\
\hline Gender (n\%) & & & 0.0132 \\
\hline Male & $350(29.3 \%)$ & 217 (35\%) & \\
\hline Female & $844(70.7 \%)$ & $403(65 \%)$ & \\
\hline Ethnicity $(\mathrm{n} \%)$ & & & 0.75 \\
\hline American Indian & $6(.5 \%)$ & $6(.97 \%)$ & \\
\hline Asian & $18(1.5 \%)$ & $7(1.1 \%)$ & \\
\hline Black or African American & $55(4.6 \%)$ & $28(4.5 \%)$ & \\
\hline White & $910(76.2 \%)$ & $468(75.5 \%)$ & \\
\hline Unreported & $205(17.2 \%)$ & $111(17.9 \%)$ & \\
\hline Body mass index (mean, SD) & $29.1(7.2)$ & $28.5(7.9)$ & 0.104 \\
\hline Periprosthetic knee fractures $(\mathrm{n} \%)$ & $363(30.4 \%)$ & $136(21.9 \%)$ & 0.0001 \\
\hline Periprosthetic hip fractures (n\%) & $831(69.6 \%)$ & $484(78.1 \%)$ & 0.0001 \\
\hline Days from admission to discharge (mean, SD) & $6.9(6.6)$ & $7.9(6.9)$ & 0.0023 \\
\hline
\end{tabular}

\section{TABLE 1: Patient demographics}

\section{Preoperative comorbidities}

Comparisons of preoperative comorbidities are detailed in Table 2. Patients who were transferred were more likely to have an ongoing malignancy $(3.6 \%$ vs. $1.1 \%, \mathrm{p}=0.03)$. In regards to ASA classification, transferred patients were more likely to have an ASA grade of $4(21.5 \%$ vs. $11.8 \%, p=0.0001)$, while not statistically significant transferred patients were more likely to have heart failure ( $11.4 \%$ vs. $8.1 \%, \mathrm{p}=0.06)$.

Patients who did not require transfer were more likely to have an ASA grade of $1(1.0 \%$ vs. $0 \%, p=0.011)$ or 2 $(21.8 \%$ vs. $13.4 \%, \mathrm{p}=0.0001)$. There were no other statistical differences or trends observed regarding smoking status or other major preoperative comorbidities. 


\section{Cureus}

\begin{tabular}{|c|c|c|c|}
\hline Preoperative comorbidities & Non-transfers & Transfers & P-value \\
\hline Smoking history (n\%) & $130(10.9 \%)$ & 57 (9.2\%) & 0.29 \\
\hline Comorbidities present (n) & 356 & 220 & \\
\hline Diabetes (n\%) & $221(62.1 \%)$ & $123(55.9 \%)$ & 0.49 \\
\hline Heart failure (n\%) & $29(8.1 \%)$ & 25 (11.4\%) & 0.06 \\
\hline Renal failure (n\%) & $3(0.8 \%)$ & $4(1.8 \%)$ & 0.24 \\
\hline Malignancy (n\%) & $4(1.1 \%)$ & $8(3.6 \%)$ & 0.03 \\
\hline Chronic obstructive pulmonary disease (n\%) & $97(27.2 \%)$ & $60(27.3 \%)$ & 0.29 \\
\hline Ascites (n\%) & $2(0.5 \%)$ & 0 & 0.55 \\
\hline \multicolumn{4}{|l|}{ ASA classification (n\%) } \\
\hline 1 & $12(1.0 \%)$ & 0 & 0.011 \\
\hline 2 & $260(21.8 \%)$ & $83(13.4 \%)$ & 0.0001 \\
\hline 3 & $778(65.2 \%)$ & $404(65.2 \%)$ & 1 \\
\hline 4 & $141(11.8 \%)$ & $133(21.5 \%)$ & 0.0001 \\
\hline
\end{tabular}

\section{TABLE 2: Preoperative comorbidities}

\section{Preoperative laboratory values}

Comparisons of preoperative laboratory values are detailed in Table 3. Patients who required transfers tended to have a higher mean creatinine ( 1.03 vs. $0.93, \mathrm{p}=0.005)$, lower mean albumin ( 3.36 vs. $3.57, \mathrm{p}<$ 0.0001 ), lower mean hematocrit ( 32.39 vs. $34.61, \mathrm{p}<0.0001$ ), and higher mean white blood cell count (8.94 vs. 8.61, $\mathrm{p}=0.034)$. A multiple logistic regression model controlling for age, sex, and BMI demonstrated that having a lower preoperative albumin (i.e., $<3.5 \mathrm{~g} / \mathrm{dL}$ ) was a risk factor for readmission within 30 days of discharge in patients who required transfers (odds ratio $=1.29,95 \%$ confidence interval $=[1.068-1.44], p=$ 0.002).

\begin{tabular}{|c|c|c|c|}
\hline Preoperative Laboratory Values & Non-transfers & Transfers & P-value \\
\hline Creatinine (mean, SD) & $0.93(0.56)$ & $1.03(0.91)$ & 0.005 \\
\hline Albumin (mean, SD) & $3.57(0.54)$ & $3.36(0.57)$ & $<0.0001$ \\
\hline Hematocrit (mean, SD) & $34.61(5.49)$ & $32.39(5.10)$ & $<0.0001$ \\
\hline White blood cell count (mean, SD) & $8.61(3.18)$ & $8.94(3.27)$ & 0.034 \\
\hline
\end{tabular}

TABLE 3: Preoperative laboratory values

\section{Complications and readmissions}

Perioperative complications and readmissions within 30 days of discharge are detailed in Table 4. No differences were observed regarding readmission rates between the non-transferred (8.3\%) and transferred (7.9\%) patients. Transferred patients had a longer mean operative time (154.1 minutes vs. 145.9 minutes, $\mathrm{p}=$ 0.014). The major complication rate between groups was $9.6 \%$ in the non-transferred group and $11.5 \%$ in the transferred group $(\mathrm{p}=0.25$ ). No significant differences were observed between groups when comparing individual complications. 


\section{Cureus}

\begin{tabular}{|c|c|c|c|}
\hline Perioperative complications and readmissions & Non-transfers & Transfers & P-value \\
\hline Operative time in minutes (mean, SD) & $145.9(66.2)$ & $154.1(66.9)$ & 0.014 \\
\hline Readmitted within 30 days ( $\mathrm{n} \%$ ) & $99(8.3 \%)$ & $49(7.9 \%)$ & 0.86 \\
\hline Complications present (n\%) & $115(9.6 \%)$ & $71(11.5 \%)$ & 0.25 \\
\hline Pulmonary embolism (n\%) & $10(0.8 \%)$ & $6(0.9 \%)$ & 0.79 \\
\hline Myocardial infarction (n\%) & $18(1.5 \%)$ & $12(2.6 \%)$ & 0.56 \\
\hline Pneumonia (n\%) & $20(1.7 \%)$ & $16(2.6 \%)$ & 0.21 \\
\hline Cerebrovascular accident (n\%) & $3(0.3 \%)$ & $5(0.8 \%)$ & 0.13 \\
\hline Wound infection (n\%) & $22(1.8 \%)$ & $8(1.3 \%)$ & 0.44 \\
\hline Superficial infections ( $\mathrm{n} \%)$ & $15(1.3 \%)$ & $5(0.8 \%)$ & 0.75 \\
\hline Deep infections $(\mathrm{n} \%)$ & $30(2.5 \%)$ & $8(1.3 \%)$ & 0.08 \\
\hline Urinary tract infection (n\%) & $42(3.5 \%)$ & $24(3.9 \%)$ & 0.69 \\
\hline
\end{tabular}

TABLE 4: Perioperative complications and readmissions

\section{Discharge destination}

Comparisons of discharge destinations are detailed in Table 5. Patients who were transferred were less likely to be discharged home $(19.4 \%$ vs. $32.2 \%, p=0.0001)$, more likely to be transferred to hospice $(0.7 \%$ vs. $0.08 \%$, $\mathrm{p}=0.049$ ), and more likely to be transferred to be transferred to a short-term or long-term rehabilitation facility $(77.4 \%$ vs. $65.7 \%, \mathrm{p}=0.0001)$.

\begin{tabular}{|c|c|c|c|}
\hline Discharge Destination & Non-transfers & Transfers & P-value \\
\hline Against medical advice ( $\mathrm{n} \%)$ & $3(0.3 \%)$ & 0 & 0.55 \\
\hline Expired (n\%) & $21(1.8 \%)$ & $16(2.6 \%)$ & 0.29 \\
\hline Home (n\%) & $385(32.2 \%)$ & $120(19.4 \%)$ & 0.0001 \\
\hline Hospice (n\%) & $1(0.08 \%)$ & $4(0.7 \%)$ & 0.049 \\
\hline Rehabilitation facility (n\%) & $784(65.7 \%)$ & $480(77.4 \%)$ & 0.0001 \\
\hline
\end{tabular}

\section{TABLE 5: Discharge destination}

\section{Discussion}

The present study aimed to elucidate the effects, if any, on interfacility transfer in patients who sustained a periprosthetic fracture of the hip or knee. Briefly, the analysis demonstrated that transferred patients had a longer length of stay, tended to be older, and were less likely to be healthy based on the ASA classification. Additionally, transferred patients were less likely to be discharged directly home, as almost $80 \%$ required discharge to a rehabilitation facility. Although hypothesized as otherwise, there were no differences between groups regarding readmission rates or perioperative complications. Lastly, lower preoperative albumin was a risk factor for readmission within 30 days of discharge in patients who were transferred.

Most of the prior literature in orthopedic trauma has emphasized the effects of patient factors on surgical outcomes. However, there has been growing interest in the effects of the pre-hospital environment on surgical outcomes. Although a significant proportion of inappropriate orthopedic trauma transfers have been previously documented, the short-term effects of transfer status have been limited to a single study. Recently, a study used the NSQIP database to determine if transfer status was associated with increased morbidity and mortality in the geriatric population with non-periprosthetic hip fractures, and similar to our results, it also showed that transfers were associated with a significantly lower proportion of home discharges. In addition, our study demonstrated that transferred patients had a longer overall mean 
hospitalization time (7.9 days vs. 6.9 days). This may be associated with a longer time to surgery, which has been previously shown to be associated with increased mortality, readmissions, and perioperative complications. Overall, the results of the present study indicate an urgency to establish a risk stratification and transfer algorithm to optimize the outcomes in this complex patient population.

Although the literature is limited, the ASA classification has been previously shown to influence outcomes in patients transferred between facilities. A recent study by Lalonde et al. showed that patients who took more medications trended toward having a higher chance of readmission [12]. Relatedly, the present study demonstrated that ASA classification is associated with transfer status, i.e., patients who were transferred tended to have an ASA grade 4, whereas non-transferees were more likely to have an ASA grade of 1 or 2. Overall, these findings are intuitive in that they suggest less-healthy patients may require higher levels of care that are not readily available at the hospital to which the patient first presents. Future studies may consider elucidating the relationship, if any, between ASA classification and the appropriateness of patient transfers.

The present study demonstrated that lower albumin levels in transferred patients were associated with increased 30-day readmission rates. In the orthopedics literature, hypoalbuminemia has been previously shown to be associated with a longer length of hospital stay, inferior clinical outcomes, higher mortality, and more postoperative complications as compared to patients with albumin levels within the normal range [13-16]. Most recently, a study by Kishawi et al. showed that patients with lower preoperative albumin levels who underwent total joint arthroplasty had increased risks of major postoperative complications and a higher 30-day readmission rate [17]. Ryan et al. compared preoperative albumin to ASA score and showed that both were significant predictors of death, superficial infections, pneumonia, renal insufficiency, reintubation, transfusion, readmissions, and reoperations; however, hypoalbuminemia was more robust in predicting deep wound infections in total hip arthroplasty and superficial wound infections in total knee arthroplasty [15]. In the context of our results, these trends further suggest that health and nutritional status in the forms of ASA classification and preoperative albumin may be important risk stratification tools when decision-making regarding patient transfers.

The present study has limitations to consider. First, given the nature of this database study, we were unable to elucidate the complexity of the periprosthetic fractures themselves. This is important to consider, as we were unable to determine if the transfers were performed because of the patient's health care history and overall medical complexity, the complexity of the fracture itself, or a combination of the two. Second, the time between initial patient presentation at the outside hospital to transfer was not available in the NSQIP database. This may have been a confounding variable that was not accounted for in the data analysis. Third, the database was limited to complication rates within 30 days. Long-term outcomes or readmission rates were not collected.

\section{Conclusions}

Transfer centers accepting and treating periprosthetic fractures should be aware that these patients often have a longer length of stay and are less likely to be discharged home. However, the data suggests these patients are well cared for, given the similar complication and readmission rates.

\section{Additional Information}

\section{Disclosures}

Human subjects: All authors have confirmed that this study did not involve human participants or tissue. Animal subjects: All authors have confirmed that this study did not involve animal subjects or tissue. Conflicts of interest: In compliance with the ICMJE uniform disclosure form, all authors declare the following: Payment/services info: All authors have declared that no financial support was received from any organization for the submitted work. Financial relationships: All authors have declared that they have no financial relationships at present or within the previous three years with any organizations that might have an interest in the submitted work. Other relationships: All authors have declared that there are no other relationships or activities that could appear to have influenced the submitted work.

\section{References}

1. Luster J, Yanagawa FS, Bendas C, Ramirez CL, Cipolla J, Stawicki SP: Interhospital Transfers: Managing Competing Priorities While Ensuring Patient Safety. IntechOpen, London, UK; 2017. 10.5772/intechopen.72022

2. Kiss T, Bölke A, Spieth PM: Interhospital transfer of critically ill patients . Minerva Anestesiol. 2017, 83:1101-8. 10.23736/S0375-9393.17.11857-2

3. Mueller SK, Fiskio J, Schnipper J: Interhospital transfer: transfer processes and patient outcomes. J Hosp Med. 2019, 14:486-91. 10.12788/jhm.3192

4. Shah S, Xian Y, Sheng S, et al.: Use, temporal trends, and outcomes of endovascular therapy after interhospital transfer in the United States. Circulation. 2019, 139:1568-77. 10.1161/CIRCULATIONAHA.118.036509

5. Froehler MT, Saver JL, Zaidat OO, et al.: Interhospital transfer before thrombectomy is associated with 
delayed treatment and worse outcome in the stratis registry (systematic evaluation of patients treated with neurothrombectomy devices for acute ischemic stroke). Circulation. 2017, 136:2311-21.

10.1161/CIRCULATIONAHA.117.028920

6. White MJ, Sutton AG, Ritter V, Fine J, Chase L: Interfacility transfers among patients with complex chronic conditions. Hosp Pediatr. 2020, 10:114-22. 10.1542/hpeds.2019-0105

7. Broman KK, Phillips SE, Ehrenfeld JM, et al.: Identifying futile interfacility surgical transfers. Am Surg. 2017, 83:866-70.

8. O'Connell RS, Haug EC, Malasitt P, Mallu S, Satpathy J, Isaacs J, Mounasamy V: Appropriateness of patients transferred with orthopedic injuries: experience of a level I trauma center. Eur J Orthop Surg Traumatol. 2018, 28:551-4. 10.1007/s00590-018-2134-x

9. Thakur NA, Plante MJ, Kayiaros S, Reinert SE, Ehrlich MG: Inappropriate transfer of patients with orthopaedic injuries to a level I trauma center: a prospective study. J Orthop Trauma. 2010, 24:336-9. 10.1097/BOT.0b013e3181b18b89

10. Boyd JA, Gradisar IM: Total knee arthroplasty after knee arthroscopy in patients older than 50 years Orthopedics. 2016, 39:e1041-4. 10.3928/01477447-20160719-01

11. Goldfarb CA, Borrelli J Jr, Lu M, Ricci WM: A prospective evaluation of patients with isolated orthopedic injuries transferred to a level I trauma center. J Orthop Trauma. 2006, 20:613-7. 10.1097/01.bot.0000249415.47871.e5

12. Lalonde S, Wood GC: Short stay total joint arthroplasty program: patient factors predicting readmission . Can J Surg. 2019, 62:182-8. 10.1503/cjs.009218

13. Liu D, Zhu Y, Chen W, Li M, Liu S, Zhang Y: Multiple preoperative biomarkers are associated with incidence of surgical site infection following surgeries of ankle fractures. Int Wound J. 2020, 17:842-50. 10.1111/iwj.13351

14. Vora M, Sing DC, Yi PH, Cheah JW, Li X: Hypoalbuminemia is a risk factor for predicting early postoperative complications after proximal humerus fracture fixation. J Orthop. 2020, 19:106-10. 10.1016/j.jor.2019.11.022

15. Ryan SP, Politzer C, Green C, Wellman S, Bolognesi M, Seyler T: Albumin versus American Society of Anesthesiologists score: which is more predictive of complications following total joint arthroplasty?. Orthopedics. 2018, 41:354-62. 10.3928/01477447-20181010-05

16. Chung AS, Hustedt JW, Walker R, Jones C, Lowe J, Russell GV: Increasing severity of malnutrition is associated with poorer 30-day outcomes in patients undergoing hip fracture surgery. J Orthop Trauma. 2018, 32:155-60. 10.1097/BOT.0000000000001081

17. Kishawi D, Schwarzman G, Mejia A, Hussain AK, Gonzalez MH: Low preoperative albumin levels predict adverse outcomes after total joint arthroplasty. J Bone Joint Surg Am. 2020, 102:889-95. 10.2106/JBJS.19.00511 